\title{
Control and Cybernetics
}

vol. 38 (2009) No. $4 \mathrm{~A}$

\section{The Maximum Principle of optimal control: A history of ingenious ideas and missed opportunities*}

\author{
by \\ Hans Josef Pesch ${ }^{1}$ and Michael Plail ${ }^{2}$ \\ ${ }^{1}$ Chair of Mathematics in Engineering Sciences, University of Bayreuth \\ D 95440 Bayreuth, Germany \\ ${ }^{2}$ Head of BGMI, Consulting in Mathematics and Computer Science \\ Munich, D 82237 Wörthsee, Germany \\ e-mail: hans-josef.pesch@uni-bayreuth.de, m.plail@bgmi-muenchen.de
}

\begin{abstract}
On the occasion of (more than) 50 years of optimal control theory, this paper deals with the development of the maximum principle of optimal control during the early times of the Cold War, when mathematicians in the US and the USSR made every endeavor to solve minimum time interception problems, which later on became prototypes of the first optimal control problems. The paper is a short survey on the history of optimal control, a sequence of ingenious ideas and regrets of missed opportunities. The conclusions of the paper are extracted from the second author's monograph on the development of optimal control theory from its commencements until it became an independent discipline in mathematics. The present paper also includes secondary literature published thereafter.
\end{abstract}

Keywords: history of optimal control, maximum principle.

\section{The Maximum Principle, a creation of the Cold War and the germ of a new mathematical discipline}

After 50 years of optimal control, it is time to look back on the early days of optimal control. Immediately after World War II, with the beginning of the Cold War, the two superpowers, US and USSR, increased their efforts in making use of mathematicians and their mathematical theories in defense analyses, since mathematics had been recognized as useful during World War II. Therefore it is not astonishing that mathematicians in East and West almost simultaneously began to develop solution methods for problems which later became known as problems of optimal control such as, for example, minimum time interception problems for fighter aircraft.

*Submitted: February 2009; Accepted: November 2009. 
Initially, engineers attempted to tackle such problems. Due to the increased speed of aircraft, nonlinear terms no longer could be neglected. Linearisation then was not the preferred method. Engineers confined themselves to simplified models and achieved improvements step by step.

Angelo Miele (1950) achieved a solution for a special case. He investigated control problems with a bounded control firstly for aircraft, later for rockets in a series of papers from 1950 on. For special cases, he was able to derive the Euler-Lagrange equation as a first, instead of a second order differential equation, which determines the solution in the phase plane. By means of Green's Theorem, Miele resolved the difficulty that the vanishing Legendre as well as the Weierstrass function did not allow a distinction between a maximum or a minimum. Such a behaviour became later known as the singular case in the evaluation of the maximum principle. ${ }^{1}$ Miele's solution for a simplified flight path optimization problem (with the flight path angle as control variable) exhibits an early example for a bang - singular - bang switching structure (in terms of aerospace engineering: vertical zoom climb - a climb along a singular subarc-vertical dive) ${ }^{2}$

Nearly parallel to Miele's works, efforts were made also in the USSR concerning dynamic optimization, particularly in rocket launching. As early as 1946, Okhotsimsky solved the specific problem of a vertically ascending rocket to achieve a given final altitude with a minimum initial mass. ${ }^{3}$ His solution consists of a motion with maximum admissible thrust, an ascent with an optimal relation between velocity and altitude, and finally a phase with thrust turned off. ${ }^{4}$

For obvious reasons, problems of the calculus of variations were restated at that time and used for control problems. However, optimal control theory was not a direct continuation of the classical calculus of variations. The arising differential equations were not yet solvable. However, the development of digital computers from the end of World War II on, yielded hope that the numerical problems would be overcome in the near future. For more details concerning the early attempts to use the calculus of variations and increasingly detailed models for the solution of optimization problems in rocket technology, see Plail (1998), pp. 110ff.

Independently, new inspirations also came from nonlinear control theory. The global comparison of competing functions by means of digital computers was an isolated procedure. By the use of dynamic programming the elaborate comparison could be reduced a bit.

\footnotetext{
${ }^{1}$ For more details, see Plail (1998), pp. 102ff, and Miele's later references concerning trajectories for rockets cited therein.

2 The notions "bang" and "singular" will be explained later; see footnotes 21 and 27.

${ }^{3}$ This problem was posed by Hamel in 1927, in distinction from the Goddard's earlier problem from 1919 of reaching a maximum altitude for a given initial mass.

${ }^{4}$ In 1957 Okhotsimsky and Eneyev investigated the optimal launch of the first Sputnik satellite.
} 
Mathematicians have attempted to treat optimal control problems with a certain delay. They pursued new ideas. When the connections and differences of their theories and methods to the classical calculus of variations became clear, ${ }^{5}$ one began to develop a unitary theory of optimal control taking into account its new characteristics. A new mathematical discipline was born.

The elaboration of the following outline of the early history of optimal control theory is based on the comprehensive monograph of Plail (1998) on the history of the theory of optimal control from its commencements until it became an independent discipline in mathematics. Since this book is written in German, it may not be widely known (an English translation is intended).

Most of the other literature concerning the historical development of optimal control theory so far are either short notes on specific aspects or autobiographical notes from scientists, who were involved in the development. For more references to secondary literature, see the book of Plail (1998). New secondary literature not incorporated in this book is now taken into account, too.

Arthur E. Bryson Jr. (1996), also one of the pioneers of optimal control, gave a survey of, mainly, the US contributions to optimal control from 1950 to 1985, particularly from the viewpoint of an aerospace engineer. This paper also includes some of the early developments of numerical methods of optimal control as well as the treatment of inequality constraints from the 1960s to the 1980s.

In 2005 Krotov and Kurzhanski gave - as they said - a subjectively flavoured retrospective to control theory from the aerospace perspective with special focus on eastern authors. They also mention the early Russian achievements in the development of numerical methods, as well as especially significant attainments after 1970 in developing new variational methods for nonsmooth or degenerate problems and in control synthesis under uncertainties.

The tongue-in-cheek article of Sussmann and Willems (1997) on "300 years of optimal control" from Johann Bernoulli's brachistochrone problem of 1696 to the maximum principle of the 1950s describes, in a unique instructive way, a fictitious development of optimal control theory out of the development of the calculus of variations by constantly asking "what if". This enlightens the connections between those closely related fields.

\section{The Maximum Principle in the US: captured by the calculus of variations and hidden by secrecy}

A first formulation and the distinction between controls and states. After the end of World War II, the RAND corporation (Research ANd Development) was set up by the United States Army Air Forces at Santa Monica, California, as a nonprofit think tank focusing on global policy issues to offer

\footnotetext{
${ }^{5}$ See Berkovitz (1961) for one of the first papers on that.
} 

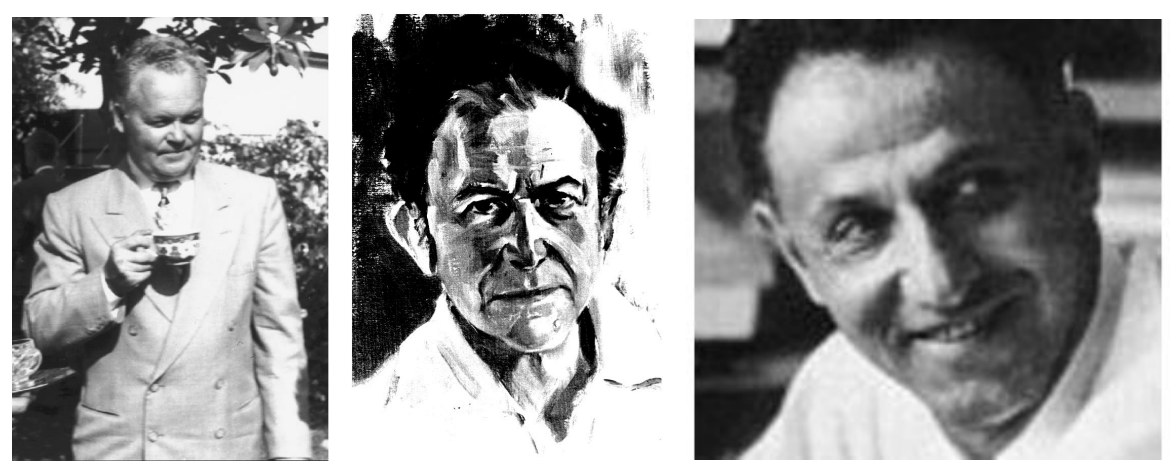

Figure 1. The mathematicians at RAND: Magnus R. Hestenes, Rufus P. Isaacs, and Richard E. Bellman

research and analysis to the United States armed forces. ${ }^{6}$ Around the turn of the decades in 1950 and thereafter, RAND simultaneously employed three great mathematicians of special interest, partly at the same time: Magnus R. Hestenes (1906-1991), Rufus P. Isaacs (1914-1981), and Richard E. Bellman (1920-1984). ${ }^{7}$ We firstly turn towards Hestenes.

Around 1950, Hestenes simultaneously wrote his two famous RAND research memoranda No. 100 and 102; see Hestenes (1949, 1950). In these reports, Hestenes developed a guideline for the numerical computation of minimum time trajectories for aircraft in the advent of digital computers. In particular, Hestenes' memorandum RM-100 includes an early formulation of what later became known as the maximum principle: the optimal control vector $a_{h}$ (angle of attack and bank angle) has to be chosen in such a way that it maximizes the Hamiltonian $H$ along a minimizing curve $C_{0}$. In his report, we already find the clear formalism of optimal control problems with its separation into state and control variables.

The starting point was a concrete optimal control problem from aerospace engineering: in Hestenes' notation, the equations of motion are given by

$$
\begin{aligned}
& \frac{\mathrm{d}}{\mathrm{d} t}(m \vec{v})=\vec{T}+\vec{L}+\vec{D}+\vec{W}, \\
& \frac{\mathrm{d} w}{\mathrm{~d} t}=\dot{W}(v, T, h),
\end{aligned}
$$

where the lift vector $\vec{L}$ and the drag vector $\vec{D}$ are known functions of the angle of attack $\alpha$ and the bank angle $\beta$. The weight vector $\vec{W}$ has the length $w$. The thrust vector $T$ is represented as a function of velocity $v=|\vec{v}|$ and altitude $h$.

\footnotetext{
${ }^{6}$ For more information on RAND, see Plail (1998), pp. 53ff.

${ }^{7}$ Hestenes has worked for RAND in 1948-1952, Isaacs in 1948-1954/55, and Bellman temporarily in 1948 and 1949, and as salaried employee in 1952-1965.
} 
Then the trajectory is completely determined by the initial values of the position vector $\vec{r}$, the velocity vector $\vec{v}$ and the norm $w$ of $\vec{W}$, as well as by the values of $\alpha(t)$ and $\beta(t)$ along the path.

The task consists of determining the functions $\alpha(t)$ and $\beta(t), t_{1} \leq t \leq t_{2}$, in such a way that the flight time $t_{2}$ is minimized with respect to all paths which have prescribed initial and terminal conditions for $\vec{r}\left(t_{1}\right), \vec{v}\left(t_{1}\right), w\left(t_{1}\right)$, $\vec{r}\left(t_{2}\right), \vec{v}\left(t_{2}\right)$, and $w\left(t_{2}\right)$.

In general formulation: consider a class of functions and a set of parameters

$$
a_{h}(t) \quad \text { and } \quad b_{\rho} \quad(h=1, \ldots, m ; \rho=1, \ldots, r)
$$

as well as a class of arcs

$$
q_{i}(t) \quad\left(t_{1} \leq t \leq t_{2} ; i=1, \ldots, n\right)
$$

which are related to each other by ${ }^{8}$

$$
q_{i}^{\prime}=\dot{Q}_{i}(t, q, a)
$$

and the terminal conditions

$$
\begin{aligned}
t_{1} & =T_{1}(b), & q_{i}\left(t_{1}\right) & =Q_{i 1}(b), \\
t_{2} & =T_{2}(b), & q_{i}\left(t_{2}\right) & =Q_{i 2}(b) .
\end{aligned}
$$

The quantities $a_{h}, b_{\rho}$, and $q_{i}$ are to be determined in such way that the functional

$$
I=g(b)+\int_{t_{1}}^{t_{2}} L(t, q, a) \mathrm{d} t
$$

is minimized. This type of optimal control problem was called Type A by Hestenes. It was not consistent with the usual formulation of problems of the calculus of variations at that time, as formulated, e. g., by Bolza (1904).

Already in 1941, Hestenes mentioned a paper of Marston Morse (1931) ${ }^{9}$ as a reference for the introduction of parameters $a_{h}$ in the calculus of variations and their transformation via $a_{h}^{\prime}(x)=0$ into the Bolza form; see Plail (1998), p. $83 .{ }^{10}$

The Bolza form was called Type B by Hestenes and was opposed to Type A: consider a class of numbers, respectively functions

$$
b_{\rho}, \quad x_{j}(t) \quad\left(t_{1} \leq t \leq t_{2} ; \rho=1, \ldots, r ; j=1, \ldots, p\right),
$$

\footnotetext{
${ }^{8}$ Here and in the following, $Q(\cdot)$, respectively $\dot{Q}(\cdot, \cdot, \cdot)$ denote the given boundary conditions at the initial and terminal point, which may depend on the design parameters $b_{\rho}$, respectively the vector field defining the right hand side of the state equations.

${ }^{9} \mathrm{M}$. Morse as well as G. C. Evans, both former AMS presidents, initiated the foundation of the War Preparedness Committee of the American Mathematical Society (AMS) and the Mathematical Association of America (MAA). More on the importance and the role of applied mathematics during World War II can be found in Plail (1998), pp. 50ff.

${ }^{10}$ Also Rufus P. Isaacs (1950) distinguished between navigation (control) and kinetic (state) variables; see also Breitner (2005), p. 532. Later Rudolf E. Kálmán as well introduced the concept of state and control variables; see Bryson (1996), p. 27.
} 
where only those curves are of interest, which satisfy the system of differential equations

$$
\phi_{i}\left(t, x, x^{\prime}\right)=0 \quad(i=1, \ldots, n<\rho)
$$

and the terminal conditions

$$
\begin{aligned}
& t_{1}=t_{1}(b), \quad x_{i}\left(t_{1}\right)=X_{i 1}(b), \\
& t_{2}=t_{2}(b), \quad x_{i}\left(t_{2}\right)=X_{i 2}(b) .
\end{aligned}
$$

The function to be minimized has the form

$$
I=g(b)+\int_{t_{1}}^{t_{2}} f\left(t, x, x^{\prime}\right) \mathrm{d} t .
$$

One can easily transform Type A into Type B by letting $x_{n+h}(t)=\int_{t_{1}}^{t} a_{h}(\tau) \mathrm{d} \tau$ and vice versa by $\phi_{n+h}\left(t, x, x^{\prime}\right)=a_{h}$; see Hestenes (1950), pp. $5 \mathrm{ff}$, respectively Plail (1998), pp. 81ff.

Hestenes then applied the known results of the calculus of variations for Type A on Type B under the usual assumptions of variational calculus such as variations belonging to open sets and state functions being piecewise continuous.

Denoting the Lagrange multipliers by $p_{i}(t)$ and introducing the function

$$
H(t, q, p, a):=p_{i} \dot{Q}_{i}-L,
$$

Hestenes obtained the first necessary condition: on a minimizing curve $C_{0}$, there must hold

$$
q_{i}^{\prime}=H_{p_{i}}, \quad p_{i}^{\prime}=-H_{q_{i}}, \quad H_{a_{h}}=0,
$$

hence also

$$
\frac{\mathrm{d}}{\mathrm{d} t} H=H_{t} .
$$

Moreover, the final values of $C_{0}$ have to satisfy certain transversality conditions, which are omitted here.

The second necessary condition derives from Weierstrass' necessary condition: along the curve $C_{0}$, the inequality

$$
H(t, q, p, A) \leq H(t, q, p, a)
$$

must hold for every admissible elements $(t, q, A)$, where a denotes a (locally) optimal control. This is a first formulation of a maximum principle, in which the variables were clearly distinguished, and which later were denoted as state, adjoint, and control variables. ${ }^{11}$

\footnotetext{
${ }^{11}$ Here, Gamkrelidze is mistaken in his historical review (1999), p. 439, on the discovery of the maximum principle. He attributes the first appearance of the adjoint system and the Hamiltonian lift of the initial families of vector fields on the state space to its cotangent bundle, the phase space of the problem, to the early works of the group around Pontryagin.
} 
The third necessary condition was the one of Clebsch (Legendre): at each element $(t, q, p, a)$ of $C_{0}$, the inequality

$$
H_{a_{h} a_{k}} \pi_{h} \pi_{k} \leq 0
$$

must hold for arbitrary real numbers $\pi_{\diamond}$.

Since both control variables appear nonlinearly in the equations of motion, Hestenes' trajectory optimization problem (Type A) can easily be interpreted as a Bolza problem of the calculus of variations.

Moreover, Hestenes also investigated optimal control problems with additional constraints in the form of

$$
\phi_{\sigma}(t, q, a)=0, \text { respectively } \phi_{\sigma}(t, q, a) \geq 0 \quad(\sigma=1, \ldots, l \leq m) ;
$$

see Hestenes (1950), pp. 29ff.

Six years before the works at the Steklov Institute in Moscow began, the achievement of introducing a formulation that later became known as the general control problem was adduced by Hestenes in his Report RM-100. This often has been credited with Pontryagin (see Section 3). Hestenes' report was considered to be hardly distributed outside RAND. However, there existed many contacts between staff members of RAND engaged in optimal control and those "optimizers" outside RAND. Therefore, the content of RM-100 cannot be discarded as a flower that was hidden in the shade [as "Schattengewächs" (nightshade plant); see Plail (1998), p. 84]. ${ }^{12}$

Hestenes was a descendant of Oskar Bolza (1857-1942) and Gilbert Ames Bliss (1876-1951) from the famous Chicago School on the Calculus of Variations. Bolza, a student of Felix Christian Klein (1849-1925) and Karl Theodor Wilhelm Weierstrass (1815-1897), had attended Weierstrass' famous 1879 lecture course on the calculus of variations. This course might have had a lasting effect on the direction that Bolza's mathematical interests have taken and that he has passed on to his descendants. In this tradition, Hestenes' derivation of his maximum principle fully relied on Weierstrass' necessary condition (and the Euler-Lagrange equation), in which the control functions are assumed to be continuous and to have values in an open control domain. These assumptions were natural for Hestenes' illustrative example of minimum time interception, but have obfuscated the potential of this principle.

Further concerns dealt with the fact that, as for example in aerospace engineering, the admissible controls cannot be assumed to lie always in open sets.

\footnotetext{
${ }^{12}$ The different circulation of Hestenes' RM-100 compared to Isaacs' RM-257, 1391, 1399, 1411, and 1486, the latter are all cited, e. g., in Breitner (2005), may have been caused by the fact that Hestenes' memorandum contains instructions for engineers while Isaacs' memoranda were considered to be cryptic. On this: Wendell H. Fleming, a colleague of Isaacs at RAND, on the occasion of the bestowal of the Isaacs Award by the International Society of Dynamic Games in July 2006: One criticism made of Isaacs' work was that it was not mathematically rigorous. He worked in the spirit of such great applied mathematicians as Laplace, producing dramatically new ideas which are fundamentally correct without rigorous mathematical proofs; see www-sop.inria.fr/coprin/Congress/ISDG06/Abstract/FlemingIsaacs.pdf.
} 
The optimal controls may also run partly along the boundaries of those sets. This kind of problems were solved with short delay in the USSR, independently from early efforts of optimal trajectories on closed sets by Bolza (1909), p. 392, and Valentine (1937). ${ }^{13}$

\section{The Maximum Principle in the USSR: liberated from the calculus of variations}

A genius and his co-workers, first proofs, cooperations and conflicts, honors and priority arguments. Lev Semyonovich Pontryagin (1908-1988), already a leading mathematician on the field of topology due to his book Topological Groups published in 1938, decided to change his research interests radically towards applied mathematics around 1952. He was additionally assured by the fact that new serendipities in topology by the French mathematicians Leray, Serre and Cartan came to the fore. ${ }^{14}$ In addition, he also was forced by M. V. Keldysh, director of the department of applied mathematics of the Steklov Institute, and by the organisation of the communist party at the institute to change his research direction. ${ }^{15}$ According to Pontryagin (1978), E. F. Mishchenko played a decisive role in this volte-face. They became close friends by this time. ${ }^{16}$ Contact was then made with colonel Dobrohotov, a professor of the military academy of aviation. ${ }^{17}$ In 1955, Pontryagin's group got together with members of the airforce. ${ }^{18}$ Like in the US, minimum time inter-

\footnotetext{
${ }^{13}$ The results of Bolza (1909) for problems with domain restrictions go even back to Weierstrass' famous lecture in summer 1879. See also Bliss and Underhill (1914). Note that Miele's approach mentioned earlier, although tailored for solving a specific problem only, exceeds the method of domain restrictions by unilateral variations as it was common in the calculus of variations.

${ }^{14}$ See also Gamkrelidze's historical review on Pontryagin's work on topological duality (2000).

${ }^{15}$ See Pontryagin (1978) and Boltyanskii (1994), p. 8. Gamkrelidze (2000), p. 2447, mentions Pontryagin's wish to have professional contacts with a potentially wider group of colleagues for giving up topology and devoting himself completely to applied problems of mathematics.

${ }^{16}$ Mishchenko, a student of P. S. Aleksandrov, who was the founder of the Moscow school of topology, under whom also Pontryagin began his scientific career, describes in his historical review of 2003, how he got under the influence of Pontryagin. In particular, he points out that Pontryagin was, indeed, the driving force towards applied mathematics, and he followed him into that interesting world, into which Pontryagin implicated also Boltyanskii and Gamkrelidze. Mishchenko cited Pontryagin from his autobiography (1998): I could not answer the question of what it was all needed for, the things that I was doing. The most vivid imagination could not lead me to believe that homological dimension theory would ever be of any practical use. ... I believe that if not all, then at least many mathematicians should turn to the original sources in their works, that is, to the application of mathematics. This is necessary both to justify its existence and to inject a fresh stream into research. Mishchenko later became Pontryagin's close collaborator in the fields of singular perturbed equations and (stochastic) differential games.

${ }^{17}$ See Boltyanskii (1994), p. 8.

${ }^{18}$ Letter of Yuri S. Ledyaev from the Steklov Institute to the first author from May 5, 1995. Gamkrelidze (1999), p. 437, mentioned two Air Force colonels visiting the Steklov Institute in
} 

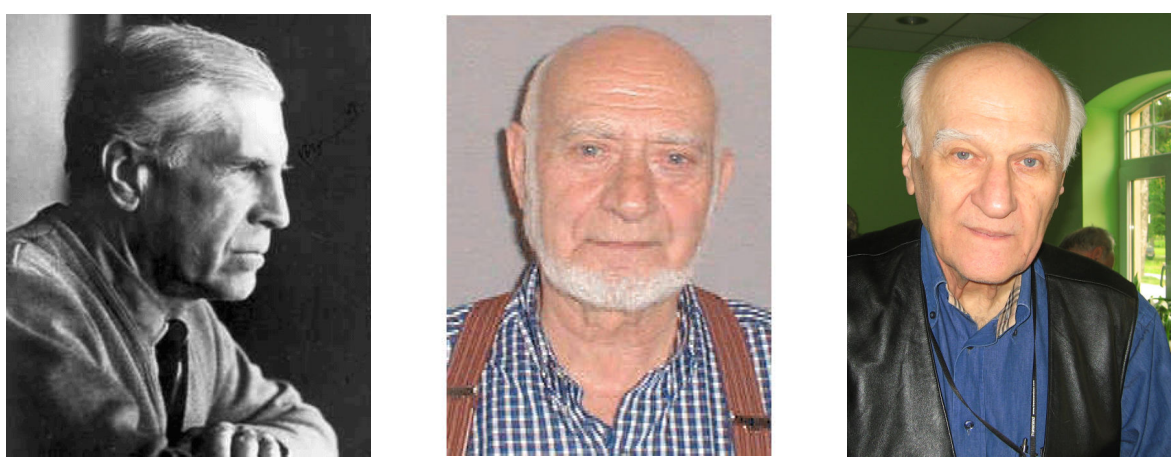

Figure 2. The mathematicians at Steklov: Lev Semyonovich Pontryagin, Vladimir Grigor'evich Boltyanskii, and Revaz Valerianovich Gamkrelidze

ception problems were tabled to Pontryagin's group; see Plail (1998), pp. 175ff.

Already prepared since 1952 by a seminar on oscillation theory and automatic control that was conducted by Pontryagin and M. A. Aizerman, a prominent specialist in automatic control, ${ }^{19}$ it was immediately clear that a time optimal control problem was at hand there. In that seminar, firstly A. A. Andronov's book on the theory of oscillations ${ }^{20}$ was studied.

However, to strengthen the applications also engineers were invited. Particularly, A. A. Fel'dbaum and A. J. Lerner focused the attention to the importance of optimal processes of linear systems for automatic control. ${ }^{21}$ Pontryagin quickly noticed that Fel'dbaum's method had to be generalized in order to solve the problems posed by the military. First results were published by Pontryagin and his co-workers Vladimir Grigor'evich Boltyanskii (born April 26, 1925) and Revaz Valerianovich Gamkrelidze (born Feb. 4, 1927) in 1956. According to Plail (1998), pp. 117ff., based on his conversation with Gamkrelidze on May 26,

the early spring of 1955. They proposed a fifth-order system of ordinary differential equations related to aircraft maneuvers with three control variables two of which entered the equations linearly and were bounded (see also Gamkrelidze, 2009, in this issue).

${ }^{19}$ See Aizerman (1958).

${ }^{20}$ See Andronov, Vitt, and Khaikin (1949). The second author of this book, A. A. Vitt, had been sent to GULag where he died. His name was forcefully removed from the first edition, but restored in the second and later editions. The GULag was the government agency that administered the penal labor camps of the Soviet Union. GULag is the Russian acronym for The Chief Administration of Corrective Labor Camps and Colonies of the NKVD, the so-called People's Commissariat for Internal Affairs, the leading secret police organization of the Soviet Union that played a major role in its system of political repression.

${ }^{21}$ In 1949 and 1955, Fel'dbaum investigated control systems of second order where the absolute value of the control has to stay on its extremum, but must change its sign once. Such a behaviour of the optimal control was later called bang-bang. Lerner (1952) generalized Fel'dbaum's results to higher order systems with several constrained coordinates, to some extent with suboptimal solutions only. For more on the evolving optimization in control theory in the USSR, see Plail (1998), pp. 163ff., and Krotov and Kurzhanski (2005). 
1994, ${ }^{22}$ the first important step was done by Pontryagin "during three sleepless nights" 23 by introducing the co-vector function $\psi$, which is uniquely determined except for a non-zero factor and satisfies the adjoint system

$$
\dot{\psi}_{i}=-\sum_{\alpha=1}^{n} \psi_{\alpha} \frac{\partial f^{\alpha}}{\partial x^{i}}(x, u) .
$$

The new method was immediately tested to be successful by means of the problems of the Bushaw-Fel'dbaum type, $\ddot{x}=u$, respectively $\ddot{x}+x=u$, and $|u| \leq 1 .^{24}$ The last inequality determines the so-called admissible set of the control values.

The early form of the maximum principle of 1956 presents itself in the following form: given the equations of motion

$$
\dot{x}^{i}=f^{i}\left(x^{1}, \ldots, x^{n}, u^{1}, \ldots, u^{r}\right)=f^{i}(x, u)
$$

and two points $\xi_{0}, \xi_{1}$ in the phase space $x^{1}, \ldots, x^{n}$, a admissible control vector $u$ is to be chosen ${ }^{25}$ in such way that the phase point passes from the position $\xi_{0}$ to $\xi_{1}$ in minimum time.

In 1956, Pontryagin and his co-workers wrote: Hence, we have obtained the special case of the following general principle, which we call maximum principle (the principle has been proven by us so far only in a series of special cases): the function

$$
H(x, \psi, u)=\psi_{\alpha} f^{\alpha}(x, u)
$$

shall have a maximum with respect to $u$ for arbitrary, fixed $x$ and $\psi$, if the vector $u$ changes in the closed domain $\bar{\Omega}$. We denote the maximum by $M(x, \psi)$. If the $2 n$-dimensional vector $(x, \psi)$ is a solution of the Hamiltonian system

$$
\begin{aligned}
& \dot{x}^{i}=f^{i}(x, u)=\frac{\partial H}{\partial \psi_{i}}, i=1, \ldots, n, \\
& \dot{\psi}_{i}=-\frac{\partial f^{\alpha}}{\partial x^{i}} \psi_{\alpha}=-\frac{\partial H}{\partial x^{i}},
\end{aligned}
$$

and if the piecewise continuous vector $u(t)$ fulfills, at any time, the condition

$$
H(x(t), \psi(t), u(t))=M(x(t), \psi(t))>0,
$$

then $u(t)$ is an optimal control and $x(t)$ is the associated, in the small, optimal

\footnotetext{
${ }^{22}$ This was reported similarly by Gamkrelidze at the Banach Center Conference on 50 Years of Optimal Control, Będlewo, Poland, on Sept. 15, 2008, too (Gamkrelidze, 2009, in this issue).

${ }^{23}$ See Gamkrelidze (1999), p. 439, and Gamkrelidze (2009), in this issue.

${ }^{24}$ See Bushaw (1952) and Fel'dbaum (1949, 1955).

${ }^{25}$ The letter $u$ stands for the Russian word for control: upravlenie; see Gamkrelidze (1999), p. 438, and Gamkrelidze (2009), in this issue.
} 
trajectory of the equations of motion. ${ }^{26,27}$ (Later on, Boltyanskii has shown that the maximum principle is only a necessary condition in the general case; according to Gamkrelidze, 1999, p. 447, it took approximately a year before a full proof of the maximum principle was found.)

After those first steps by Pontryagin, the subsequent work has been divided between Gamkrelidze and Boltyanskii. Gamkrelidze considered the second variation and achieved results equivalent to Legendre's condition. According to Gamkrelidze, ${ }^{28}$ Pontryagin made a further important step. He formulated Gamkrelidze's local maximum principle as a global (sufficient) maximum principle with respect to all admissible controls. This condition then contains no pre-conditions for the controls. The controls can take values in an arbitrary Hausdorff space and the control functions can be extended to measurable functions. $^{29}$ Gamkrelidze proved the maximum principle to be a necessary and sufficient condition in the linear case $(1957,1958)$, while Boltyanskii proved it to be "only" a necessary condition in the general case. He published the proof firstly separately (1958), later on together with Pontryagin and Gamkrelidze (1960). Boltyanskii's proof was very intricate and required substantial knowledge of the Lebesgue integral, of differential equations with measurable right hand sides, as well as of the weak compactness property of the sphere in the space of linear continuous functionals. In particular, Boltyanskii introduced needle variations, i. e. variations which are anywhere zero except for a small interval where they can take arbitrary values, as well as the separability concept of cones created by disturbances of the trajectories. ${ }^{30}$ Later Pontryagin detected

\footnotetext{
${ }^{26}$ Translated from Boltyanskii, Gamkrelidze, Pontryagin (1956) by J. H. Jones for the Technical Library, Space Technology Laboratories, Inc.

${ }^{27}$ Bang-bang and/or singular optimal controls, as mentioned in the footnotes 2 and 21 , can particularly occur if the Hamiltonian depends linearly on the control variable. In this case, isolated zeros of the so-called switching function, which is given by the factor in front of the (scalar) control $u$, determine switches between bang-bang control arcs with control values on the boundary of the admissible set (see footnote 21), whereas non-isolated zeros lead to singular control arcs (see footnote 2).

${ }^{28}$ based on the second author's conversation with Gamkrelidze on May 26, 1994, and on Gamkrelidze's talk on Sept. 15, 2008, at the Banach Center Conference on 50 Years of Optimal Control, Będlewo, Poland (Gamkrelidze, 2009, in this issue). See also Gamkrelidze's historical reviews on the discovery of the maximum principle (1999), p. 443.

${ }^{29}$ The advantages of the method of Pontryagin's group were later clearly highlighted in their book (1967), pp. $7 \mathrm{ff}$.

${ }^{30}$ Boltyanskii's proof greatly influenced the later development of the modern theory of extremum problems by A. Ya. Dubovitskii and A. A. Milyutin (1965) from the mid 1960s till the end of the 1980s. They have elaborated the relations between the calculus of variations and optimal control theory from the viewpoint of modern mathematics. In particular, they developed stationarity conditions for smooth problems with equality and inequality constraints yielding both Lagrange multiplier rules and the well-known Euler-Lagrange equations of the classical calculus of variations, which can be combined into the maximum principle. They also investigated state constrained problems as well as mixed state-control constrained problems under certain regularity assumptions yielding generalized maximum principles. Additionally, they considered mixed constraints without those regularity assumptions, known to exhibit severe difficulties because of the lack of regularity of the Lagrange multipliers. These prob-
} 
that Boltyanskii's variations have already been introduced by Edward James McShane (1904-1989) for intrinsic mathematical reasons. By means of these variations, McShane (1939) was able to prove a Lagrange multiplier rule in the calculus of variations for the Weierstrass condition in which he could abstain from the distinction between normal and abnormal curves. He could not suspect how important this would become later in the theory of optimal control.

Prior to the appearance of this paper, the Weierstrass condition could only be established under the assumption that the Euler equations satisfy a condition called normality. This condition is not verifiable a priori. In the aforementioned paper, McShane established the Weierstrass condition without assuming normality. The proof was novel: firstly, because of constructing a convex cone generated by first-order approximations to the end points of perturbations of the optimal trajectory and, secondly, because of showing that optimality implies that this cone and a certain ray can be separated by a hyperplane. McShane (1978) wrote: The research of the Chicago School on the calculus of variations was a part of pure mathematics and, in contrast, the development in optimal control emerged from practical applications. In pure mathematics, questions have been answered that nobody has posed; see Plail (1998), p. 17.

The research efforts at the Steklov Institute led to a series of publications, some of which have been already mentioned, e. g., Boltyanskii (1958), Gamkrelidze (1957-1960), ${ }^{31}$ Pontryagin $(1959,1960)$, and together in 1960 (Boltyanskii, Gamkrelidze and Pontryagin, 1960) some of which were promptly translated into English, and culminated in the famous book of Pontryagin, Boltyanskii, Gamkrelidze and Mishchenko (1961), which is a standard work of optimal control theory until today. This book has accounted for the broadening of separation theorems in proofs of optimal control, nonlinear programming, and abstract optimization theory. In 1962, Pontryagin, Boltyanskii, Gamkrelidze and Mishchenko received the Lenin prize for their work. ${ }^{32}, 33$

lems lead to families of maximum principles. See Dmitruk (1993) for more details and the associated references therein.

Also in Mordukhovich's books (2006) on variational analysis, being the subsumption of the classical variational calculus, of optimal control theory and mathematical programming, extensive comments on the early and later history of extremum problems can be found in Chapters $6.5 .1,15,21-26$ of Vol. II.

${ }^{31}$ In his book of 1960 , Gamkrelidze discovered a new proof of the maximum principle not based on needle variations but on chattering controls.

${ }^{32}$ In his historical review (2003), Mishchenko describes both the outstanding contribution of the summit of the development of the Pontryagin-Gamkrelidze-Boltyanskii mathematical theory of optimal control (in the chronological order of the early stages) and his joint paper with Pontryagin on the study of singularly perturbed differential equations exhibiting periodic motions containing alternating portions of slow phase variations and rapid, almost instantaneous ones, called relaxation oscillations. Their approximate computation draw the attention of Pontryagin and Mishchenko, in particular the study of bifurcations in neigborhoods of points where there is a jump. Their 1955 paper (Mishchenko and Pontryagin, 1955) was attached to the nomination for the Lenin Prize as suggested by Kolmogorov.

${ }^{33}$ N. N. Krasovskii, another participant of the famous Pontryagin-Aizerman seminar see the acknowledgment in Krasovskii (1957b), developed, in a series of papers from 1957 
In addition, Pontryagin also got the opportunity to present the results of his group on international conferences. For example, at the International Congress of Mathematicians in Edinburgh in August 1958, shortly after he had been elected a member of the Russian Academy of Sciences. At this time, the proof of the maximum principle was already completely elaborated. This conference was followed by the first congress of the International Federation of Automatic Control in 1960 in Moscow, where also the relations between the maximum principle and the calculus of variations were discussed.

Both Boltyanskii and Gamkrelidze coincide in their statements as to the authorship inasmuch as the comparable conditions of the calculus of variations were not known during the development phase of the maximum principle, although Bliss' monograph of 1946 existed in a Russian translation from 1950.

At that time, scientific publications became soon mutually known; see Plail (1998), pp. 182ff, for more details. In particular, McShane's needle variations could have encroached upon the proof of the maximum principle. According to Boltyanskii, ${ }^{34}$ he came across McShane's ideas a few weeks after the decisive ideas for his proof. Boltyanskii claimed the version of the maximum principle as a necessary condition to be his own contribution and described how Pontryagin hampered his publication. He said Pontryagin intended to publish the results under the name of four authors. After Boltyanskii refused to do so, he was allowed to publish his results in 1958 but said that he had to appreciate Pontryagin's contribution disproportionally. Moreover, he had to refer to McShane's needle variations and to call the principle Pontryagin's maximum principle. ${ }^{35}$

This priority argument may be based on the fact that Pontryagin wanted to head for a globally sufficient condition after Gamkrelidze's proof of a locally sufficient condition, and not to a necessary condition as it turned out to be after Boltyanskii's proof. Boltyanskii may have felt very uncomfortable to write in his monograph (1969), p. 5: The maximum principle was articulated as hypothesis by Pontryagin. Herewith he gave the decisive impetus for the development of the theory of optimal processes. Therefore the theorem in question

onwards, a distinctive approach for the solution of optimal programming controls by means of functional analytical methods. For the linear case, he embedded the optimal control problem into a well-elaborated momentum problem and revealed the minimum property of trajectories of the adjoint system in the maximum principle. In particular, Krasovskii pointed the way to the missing information for the boundary conditions in the maximum principle; compare Berdyshev et al. (2004). Results on linear control systems can also be found in the western literature; see Plail's elaboration (1998), pp. 149-151, 161, 162, on the works of R. Bellman, I. Glicksberg, and O. Gross, and, in particular, of J. P. La Salle.

${ }^{34}$ See Boltyanskii (1994).

${ }^{35}$ According to Boltyanskii, Rozonoér (1959) was encouraged to publish a tripartite work on the maximum principle in Avtomatika $i$ Telemekhanika, in order to disseminate the knowledge of the maximum principle in engineering circles and to contribute thereby to the honour of Pontryagin as discoverer of the maximum principle. Felix Chernousko called the authors' attention to the fact that Rozonoér gave a correct proof of the maximum principle for the nonlinear case, though for the simpler case of unprescribed final points, hence not so general as Boltyanskii's (talk with the second author on Jan. 26, 1994). 
and the closely related statements are called Pontryagin's maximum principle in the entire world - and rightly so. Nevertheless, Boltyanskii felt suppressed and betrayed of the international recognition of his achievements. After the end of the USSR, Boltyanskii was able to extend his fight for the deserved recognition of his work. Hence the role of Pontryagin is differently described by Boltyanskii and Gamkrelidze, both under the influence of strong personal motivations. ${ }^{36}$ For more on this, see Plail (1998), pp. 186ff.

Pontryagin received many honours for his work. He was elected member of the Academy of Sciences in 1939, and became a full member in 1959. In 1941 he was one of the first recipients of the Stalin prize (later called the State Prize). He was honoured in 1970 by being elected Vice-President of the International Mathematical Union.

\section{Regretted and claimed "missed opportunities"}

Going back to the antiquity of the development of the maximum principle, one inevitably comes across with Carathéodory's work. Variational problems with implicit ordinary differential equations as side conditions have already been investigated intensively by Constantin Carathéodory (1873-1950) in 1926; see also his book of 1935, pp. 347ff. In these publications, one can find both the identification of the degrees of freedom for the optimization by a separate notation (in Carathéodory's work by different arrays of indices), ${ }^{37}$ and a representation of Weierstrass' necessary conditions via the Hamiltonian. ${ }^{38}$ Carathéodory clearly pointed to the fact that all necessary conditions of the calculus of variations can be expressed via the Hamiltonian. Hence, the Hamiltonian formalism plays an important role on Carathéodory's famous "Royal Road of the Calculus of Variations". ${ }^{39}$

A few years later Lawrence M. Graves $(1932,1933)$ developed equivalent results for Volterra integral equations that are known to comprehend initial value problems for explicit ordinary differential equations. Graves distinguished the state variables and the degrees of freedom by different letters. ${ }^{40}$ Both results

\footnotetext{
${ }^{36}$ Compare Boltyanskii (1994) and Gamkrelidze (1999, 2000).

${ }^{37}$ See Carathéodory (1926), p. 203, formula (15) or Carathéodory (1935), p. 352, formula (425.1).

${ }^{38}$ See Carathéodory (1926), pp. 207ff., formulae $(24,29,30)$, respectively pp. 221ff., formulae $(66,72,73)$ or Carathéodory (1935), p. 358, formula (431.7).

${ }^{39}$ For more details, in particular on the impact of Carathéodory's work on optimal control, see Pesch and Bulirsch (1994).

${ }^{40}$ In 1978, William T. Reid claimed that the earliest place in the literature of the calculus of variations and optimal control theory wherein one finds a "Weierstrass condition" or "extremum principle" for a problem of Lagrange or Bolza type in "control formulation" is a 1933 paper by L. M. Graves.

Reid as well as the mathematicians at RAND and the Pontryagin group overlooked, respectively did not recognize Carathéodory's earlier works. W. H. Fleming in Breitner (2005), p. 540: In the context of calculus of variations, both dynamic programming and a principle of optimality are implicit in Carathéodory's earlier work, which Bellman overlooked.
} 
have been accomplished prior to Hestenes. However, neither the different roles of the functions, that later were labeled as state, respectively control, variables were clearly characterized nor a local maximum principle solely with respect to the controls was stated, and not at all the global maximum principle of Pontryagin's group. Nevertheless, Carathéodory's and Graves' form of the Weierstrass condition can both be considered as precursors of the maximum principle.

Simultaneously with the treatment of optimal control problems, the first differential games were established at RAND, too. Indeed, problems of optimal control can be regarded as a special case of differential games, today preferably denoted as dynamic games. Undoubtedly, Isaacs is a precursor in this field.

Isaacs was particularly working on continuous pursuit-evasion games in the early 1950s. Here, two players try to optimize a given payoff-function subject to a given dynamical system described by ordinary differential equations, the one player by minimizing, the other by maximizing it. That is a two-sided optimal control problem or, seen from the other point of view, an optimal control problem is a one-player differential game. ${ }^{41}$

Also in the early 1950s, Richard Bellman worked at RAND on multi-stage decision problems, such as optimal allocation and bottle-neck problems. Extending Bellman's principle of optimality, it is possible to derive a form of a maximum principle; see, e. g., Plail (1998), pp. $118 \mathrm{ff}$ and $216 .{ }^{42}$

In the middle of the 1960s, relations between the calculus of variations, the maximum principle and the dynamic programming were clearly recognized and described. Therefore, it is not astonishing that Hestenes, Bellman, as well as Isaacs have regretted their "missed opportunities". ${ }^{43}$

Bellman (1984) wrote: I should have seen the application of dynamic programming to control theory several years before. I should have, but I did not. One of Bellman's achievements is his criticism of the calculus of variations because of the impossibility of solving the resulting two-point boundary-value problems for nonlinear differential equations at that time. Moreover, he wrote: The tool we used was the calculus of variations. What we found was that very

Here the authors do not agree. Dynamic programming is solely Bellman's contribution whereas the principle of optimality can already be found in Jacob Bernoulli's reply of 1697 to his brother's Johann posed challenge of 1696 for the curve of quickest descend; see Pesch and Bulirsch (1994).

${ }^{41}$ A large collection of early papers on dynamic games including Isaacs' famous RAND reports RM-257, 1391, 1399, 1411, and 1486 can by found in Breitner (2005). For more on the history of differential games, in particular to the homicidal chauffeur game, we refer to Patsko and Turova (2008).

${ }^{42}$ Note that the so-called Bellman equation, also known as Hamilton-Jacobi-Bellman equation, on which Bellman's principle of dynamic programming is based, is due to Carathéodory (1935), p. 349; see also Pesch and Bulirsch (1994).

${ }^{43}$ During the historical session at the Banach Center Conference on 50 Years of Optimal Control organized by Alexander Ioffe, Kazimierz Malanowski and Fredi Tröltzsch in Będlewo, Poland, on Sept. 15, 2008, Revaz Valerianovich Gamkrelidze said: My life was a series of missed opportunities, but one opportunity, I have not missed, was to have met Pontryagin. This was a part of some longer discussions about "missed opportunities". 
simple problems required great ingenuity. A small change in the problem caused a great change in the solution. ... As soon as we turn to the numerical solution of two-point boundary-value problems for nonlinear differential equations the circumstances change abruptly.

Also Isaacs later complained with respect to his "tenet of transition" [see Isaacs $(1951,1965)]$ : Once I felt that here was the heart of the subject ... Later I felt that it ... was a mere truism. Thus in (my book) "Differential Games" it is mentioned only by title. This I regret. I had no idea, that Pontryagin's principle and Bellman's maximal principle (a special case of the tenet, appearing little later in the RAND seminars) would enjoy such widespread citation. See Isaacs (1973), p. 20. Indeed, even Isaacs' tenet represents a minimaximum principle. ${ }^{44}$ However, he had the greatness to understand: The history of mathematics has often shown parallel evolution when time was ripe. ${ }^{45}$

Both looked back at their "missed opportunities". However, Hestenes claimed that he had seen the significance of the Weierstrass condition already in his report RM-100: It turns out that I had formulated what is now known as the general optimal control problem. I wrote it up as a RAND report and it was widely circulated among engineers. I had intended to rewrite the results for publication elsewhere and did so about 15 years later. ${ }^{46}$ As a reason for the delay, he mentioned his workload as chairman at the University of Southern California and his duties at the Institute for Numerical Analysis.

More important are Hestenes' meritoriousnesses. Hestenes indeed expressed Weierstrass' necessary condition as a maximum principle for the Hamiltonian. Herewith he had observed the importance of Weierstrass' condition for the theory of optimal control. However, he did not try to unhinge that condition from the environment of the calculus of variations and to generalize it. There is no evidence for a proof of a general form of the maximum principle.

Additionally, Hestenes brought certain problems of the calculus of variations into a form by introducing control variables, which was more appropriate for control problems. His model for the determination of "paths of least time" for aircraft already consisted of all components for a realistic three-dimensional flight path optimization problem that even included a control-state-variable inequality constraint. All this was buried in a RAND report and did not attract attention, so Boltyanskii (1994). ${ }^{47}$ Hestenes' achievement for the standardization of control problems must therefore not be underestimated; see Plail (1998), p. 188 .

\footnotetext{
${ }^{44}$ Compare the reference to Fleming in footnote 12.

${ }^{45}$ cited in Breitner (2005), p. 542, from Isaacs (1975).

${ }^{46}$ Hestenes in a letter to Saunders MacLane, printed in MacLane (1988).

${ }^{47}$ This has also been affirmed by Isaacs concerning his RAND reports; see Breitner (2005), p. 540. Compare, however, also footnote 12 concerning the varying number of copies of the RAND memoranda.
} 


\section{Conclusions}

Around the turn of the decades in 1950, Magnus R. Hestenes made the first decisive steps at the US RAND corporation with a new innovative formulation for Bolza type problems perfectly suited for the new class of problems that were under parallel investigations in East and West. This class later became known as optimal control problems. He has definitely introduced different notations for the state and the control variables, which must not be underestimated. Therewith, he was able to formulate a first maximum principle. Standing in the tradition of the famous Chicago School on the Calculus of Variations, he, however, was captured by the too strong assumptions which were common in variational calculus at that time.

In a parallel, probably independent development at RAND, Rufus P. Isaacs and Richard E. Bellman have obtained results where a maximum principle was hidden, too, without recognizing the consequences and the interconnections between their research fully. None of these three RAND fellows gave a rigorous proof for a general maximum principle at that time.

As recently as in the mid 1950s, a group around Lev S. Pontryagin at the USSR Steklov Institute made the crucial steps. Starting from the scratch, they could break new grounds. Pontryagin had the first ideas, gave the impetus and the guidelines that his co-workers Revaz V. Gamkrelidze and Vladimir G. Boltyanskii had to fill. They succeeded in doing the first proofs for a general maximum principle in the linear, respectively nonlinear case. These achievements have enabled new theoretical and practical methods for the solution of a class of problems which was called optimal processes, respectively optimal control problems. Herewith a new field in mathematics was born which is still growing (in particular with respect to constraints in form of partial differential equations) despite its accomplished theoretical maturity and its potential for solving problems of enormous complexity and of utmost practical importance today (in particular with respect to constraints in form of ordinary differential equations).

Nevertheless, one also has to appreciate Carathéodory's work as well as the contributions of Graves which flashed on the approach of a new field in mathematics. The time seemed to be ripe, however World War II has then stopped any further research in this direction. Nevertheless, it seems to be remarkable that neither Hestenes nor Bellmann nor the Pontryagin group ever made a reference to Carathéodory's book from 1935, respectively his publication from 1926.

Acknowledgements. The authors would like to thank John Burns, Andrei Dmitruk, Boris Mordukhovich, Subbaram Naidu, Valerii Patsko and Varvara Turova for their numerous comments and suggestions. 


\section{References}

Aizerman, M.A. (1958) Lektsii po teorii avtomaticheskogo regulirovaniya. Moscow, Fizmatgiz. Translated into English: Theory of Automatic Control. Oxford, London, Pergamon 1963.

Andronov, A.A., Vitt, A.A. and Khaikin, C.E. (1949) Teoriya kolebanii. Gosudarstvennoye Izdatel'stvo Fiziko-Matematicheskoi Literatury, Moscow. Translated into English: Theory of Oscillators. Pergamon Press, Oxford, 1966. Dover Publications, New York, 1987.

Bellman, R.E. (1954a) The Theory of Dynamic Programming. Bulletin of the American Mathematical Society 60, 503-516.

Bellman, R.E. (1954b) Dynamic Programming and a New Formalism in the Calculus of Variations. Proceedings of the National Acadademy of Sciences U.S.A. 40, 231-235.

Bellman, R. (1984) Eye of the Hurricane, an Autobiography. World Scientific Publishing, Singapore.

Berdyshev, V.I., Kurzhanski, A.B., Mishchenko, E.F., Osipov, Yu.S. and Rumyantsev, V.V. (2004) Nikolai Nikolaevich Krasovskii (On the occasion of his 80th birthday). Proceedings of the Steklov Institute of Mathematics, Suppl. 2, S1-S 19. Translated from Trudy Instituta Matematiki i Mekhaniki UrO RAN, 10 (2), 2004.

Berkovitz, L. (1961) Variational Methods in Problems of Control and Programming. Journal of Mathematical Analysis and Applications 3, 145169.

Bliss, G.A. (1946) Lectures on the Calculus of Variations. The University of Chicago Press, Chicago. Seventh edition, 1963. Translated into Russian, Moscow, 1950.

Bliss, G.A. and Underhill, A.L. (1914) The Minimum of a Definite Integral for Unilateral Variations in Space. American Mathematical Society Transactions 15, 291-310.

BoltyanskiI, V.G. (1958) The Maximum Principle in the Theory of Optimal Processes (in Russian). Doklady Akademii Nauk SSSR 119, 1070-1073.

BoltyanskiI, V.G. (1969) The Mathematical Methods of Optimal Control (in Russian). Nauka, Moscow. Translated into German. Mathematische Methoden der optimalen Steuerungen. Geest und Portig, Leipzig 1971. Carl Hanser Verlag, München, 1972.

Boltyanskit, V.G. (1994) The Maximum Principle - How it came to be? Schwerpunktprogramm der Deutschen Forschungsgemeinschaft "Anwendungsbezogene Optimierung und Steuerung", Report No. 526, University of Technology Munich, Munich.

Boltyanskit, V.G., Gamkrelidze, R.V. and Pontryagin, L.S. (1956) On the Theory of Optimal Processes (in Russian). Doklady Akademii Nauk SSSR 110, 7-10. 
Boltyanskit, V.G., Gamkrelidze, R.V. and Pontryagin, L.S. (1960) The Theory of Optimal Processes I. The Maximum Principle (in Russian). Izvestija Akademii Nauk SSSR Ser. Mat. 24, 3-42.

BolzA, O. (1904/1909) Lectures on the Calculus of Variations. University of Chicago Press, Chicago, 1904. Republished by Dover Publications, New York, 1961. Vorlesungen über Variationsrechnung. Revised and considerably extended German edition, Teubner, Leipzig, Berlin, 1909.

Breitner, M.H. (2005) The Genesis of Differential Games in Light of Isaacs' Contributions. Journal of Optimization Theory and Applications 124, No. 3, 523-559.

Bryson Jr., A.E. (1996) Optimal Control - 1950 to 1985. IEEE Control Systems Magazine 16, No. 3, 26-33.

Bushaw, D.W. (1952) Differential Equations with a Discontinuous Forcing Term. PhD thesis, Princeton University.

Carathéodory, C. (1926) Die Methode der geodätischen Äquidistanten und das Problem von Lagrange. Acta Mathematica 47, 199-236; see also Gesammelte Mathematische Schriften von Constantin Carathéodory 1 (Variationsrechnung), 212-248, 1954. Edited by the Bayerische Akademie der Wissenschaften. C. H. Beck'sche Verlagsbuchhandlung, München.

CARATHÉOdory, C. (1935) Variationsrechnung und partielle Differentialgleichungen erster Ordnung. Teubner, Leipzig. Calculus of Variations and Partial Differential Equations of the First Order, Part 1, Part 2. Holden-Day, San Francisco, California, 1965-1967.

Reprint of the German edition with contributions by H. Boerner and E. Hölder (Eds.), commented and extended by R. Klötzler. TeubnerArchiv der Mathematik 18, Teubner, Stuttgart, Leipzig, 1994.

Dmitruk, A.V. (1993) Maximum Principle for the General Optimal Control Problem with Phase and Regular Mixed Constraints. Computational Mathematics and Modeling 4, No. 4, 364-377.

Dubovitskit, A.YA. and Milyutin, A.A. (1965) Extremum Problems in the Presence of Restrictions (in Russian). Journal of Computational Mathematics and Mathematical Physics 5, 395-453.

Fel'dbaum, A.A. (1949) Jump Controls in Control Systems (in Russian). Avtomatika i Telemekhanika 10, 249-266.

FeL'DBaum, A.A. (1955) On the synthesis of Optimal Systems of Automatic Control (in Russian). Transactions of the Second National Conference on the Theory of Automatic Control 2. Izdatel'stvo Akademii Nauk SSSR, Moscow, 325-360.

Gamkrelidze, R.V. (1957) On the Theory of Optimal Processes in Linear Systems (in Russian). Doklady Akademii Nauk SSSR 116, 9-11.

Gamkrelidze, R.V. (1958) On the General Theory of Optimal Processes (in Russian). Doklady Akademii Nauk SSSR 123, 223-226. Completely translated into English in: Automation Express 2, March 1959. 
Gamkrelidze, R.V. (1959) Processes which have an Optimal Speed of Response for Bounded Phase Coordinates (in Russian). Doklady Akademii Nauk SSSR 125, 475-478. Extracts in: Automation Express 2, 1959.

Gamkrelidze, R.V. (1960) Principles of Optimal Control Theory. Plenum Press.

Gamkrelidze, R.V. (1999) Discovery of the Maximum Principle. Journal of Dynamical and Control Systems 5, No. 4, 437-451.

Gamkrelidze, R.V. (2000) The Mathematical Work of L. S. Pontryagin. Journal of Mathematical Sciences 100 (5), 2447-2457.

Gamkrelidze, R.V. (2009) Hamiltonian form of the Maximum Principle. Control and Cybernetics, this issue.

Goddard, R.H. (1919) A Method of Reaching Extreme Altitudes. Smithsonian Miscellaneous Collections 71 (2). Smithsonian Institution, Washington.

Graves, L.M. (1932) The Weierstrass Condition for the Problem of Bolza in the Calculus of Variations. Annals of Mathematics 33, 747-752.

Graves, L.M. (1933) A Transformation of the Problem of Lagrange in the Calculus of Variations. Transactions of the American Mathematical Society 35, 675-682.

Hamel, G. (1927) Über eine mit dem Problem der Rakete zusammenhängende Aufgabe der Variationsrechnung. Zeitschrift für angewandte Mathematik und Mechanik 7, 451-452.

Hestenes, M.R. (1949) Numerical Methods for Obtaining Solutions of Fixed End Point Problems in the Calculus of Variations. Research Memorandum No. 102, RAND Corporation, Santa Monica.

Hestenes, M.R. (1950) A General Problem in the Calculus of Variations with Applications to the Paths of Least Time. Research Memorandum No. 100, ASTIA Document No. AD 112382, RAND Corporation, Santa Monica.

IsAaCs, R.P. (1951) Games of Pursuit. Paper No. P-257, RAND Corporation, Santa Monica.

IsAaCs, R.P. (1965) Differential Games: A Mathematical Theory with Applications to Warfare and Pursuit, Control, and Optimization. John Wiley and Sons, New York. Revised second edition, John Wiley and Sons, New York, 1967. Translated into Russian. Mir, Moscow, 1967. Revised and extended third edition, Krieger, Huntington, 1975.

IsAacs, R.P. (1973) Some Fundamentals of Differential Games. In: A. Blaquiére, ed., Topics in Differential Games. North-Holland Publishing Company, Amsterdam, The Netherlands.

Lerner, A.YA. (1952) Improvement of the Dynamical Properties of Automatic Compensation by Nonlinear Feedback (in Russian). Avtomatika $i$ Telemekhanika 13, 134-144, 429-444.

Krasovskit, N.N. (1957a) On a Problem of Optimal Regulation (in Russian). Prikladnaya Matematika i Mekhanika 21 (5), 670-677. 
Krasovskit, N.N. (1957b) On the Theory of Optimal Regulation (in Russian). Avtomatika i Telemekhanika 18 (1), 960-970.

Krotov, V.F. and Kurzhanski, A.B. (2005) National Achievements in Control Theory (The Aerospace Perspective). Annual Reviews in Control 29 (1), 13-31.

MacLane, S. (1988) The Applied Mathematics Group at Columbia in World War II. In: P.L. Duren, R. Askey and U.C. Merzbach, eds., A Century of American Mathematics, Part I-III. American Mathematical Society, Providence.

McShane, E.J. (1939) On Multipliers for Lagrange Problems. American Journal of Mathematics 61, 809-819.

MCShane, E.J. (1978) The Calculus of Variations from the Beginning to Optimal Control. In: A.B. Schwarzkopf, W.G. Kelley and S.B. Eliason, eds., Optimal Control and Differential Equations. Academic Press, New York, 2-49.

Miele, A. (1950) Problemi di Minimo Tempo nel Volo Non-Stazionario degli Aeroplani (Problems of Minimum Time in the Nonsteady Flight of Aircraft). Atti della Accademia delle Scienze di Torino, Classe di Scienze Matematiche, Fisiche e Naturali 85, 41-52.

Mishchenko, E.F. (2003) About Aleksandrov, Pontryagin and Their Scientific Schools. In: A.A. Bolibruch, Yu.S. Osipov, Ya.G. Sinai, V.I. Arnold, L.D. Faddeev, Ya.I. Manin, V.B. Philippov, V.M. Tikhomirov and A.M. Vershik, eds., Mathematical Events of the Twentieth Century. Original Russian edition published by PHASIS, Moscow. English edition published jointly with PHASIS, Moscow, 2006.

Mishchenko, E.F. and Pontryagin, L.S. (1955) Periodic Solutions of Systems of Differential Equations near to Discontinuous Ones (in Russian). Doklady Akademii Nauk SSSR 110, 889-891.

Mordukhovich, B.S. (2006) Variational Analysis and Generalized Differentiation, I: Basic Theory, II: Applications. Series Fundamental Principles of Mathematical Sciences 330, 331. Springer, Berlin.

Morse, M. (1931) Sufficient Conditions in the Problem of Lagrange with Variable End Conditions. American Journal of Mathematics 53, 517-546.

Oknotsimsky, D.E. (1946) On the Theory of Rocket Propulsion (in Russian). Prikladnaya Matematika i Mekhanika 10 (2), 251-272.

Oknotsimsky, D.E. and Eneyev, T.M. (1957) Some Variational Problems Related to the Launch of an Artificial Satellite of the Earth (in Russian). Uspekhi Fizicheskikh Nauk 63 (1).

Patsko, V.S. and Turova, V.L. (2008) Homicidal Chauffeur Game: History and Modern Studies. Scientific Report. Ural Branch of the Russian Academy of Sciences, Institute of Mathematics and Mechanics, Ekaterinburg. Annals of the International Society of Dynamic Games, to appear. 
Pesch, H.J., Bulirsch, R. (1994) The Maximum Principle, Bellman's Equation and Carathéodory's Work. Journal of Optimization Theory and Applications 80 (2), 203-229.

Plail, M. (1998) Die Entwicklung der optimalen Steuerungen. Vandenhoeck \& Ruprecht, Göttingen.

Pontryagin, L.S. (1959) Optimal Control Processes II (in Russian). Uspekhi Matematicheskikh Nauk 14, 3-20. Translated into English in: Automation Express 2, July 1959. (Part I in Automation Express 1.)

Pontryagin, L.S. (1960) Optimal Processes of Regulation. Proceedings of the International Mathematical Congress, Edinburgh, August 14-21, 1958. Cambridge University Press.

Pontryagin, L.S. (1978) A Short Autobiography of L. S. Pontryagin (in Russian). Uspekhi Matematicheskikh Nauk 33, 7-21. Russian Mathematical Surveys 33, 7-24.

Pontryagin, L.S. (1998) The Life of Lev Semenovich Pontryagin, Mathematician (in Russian). Prima V, Private Publisher.

Pontryagin, L.S., Boltyanskit, V.G., Gamkrelidze, R.V. and MishCHENKo, E.F. (1961) Matematicheskaya teoriya optimal 'nykh prozessov. Fizmatgiz, Moscow. Translated into English. The Mathematical Theory of Optimal Processes. John Wiley and Sons (Interscience Publishers), New York, 1962. Translated into German. Mathematische Theorie optimaler Prozesse. Akademie-Verlag, Leipzig, 1964. Second revised German edition, Oldenbourg, München, 1967.

ReID, W.T. (1978) A Historical Note on the Maximum Principle. SIAM Review 20 (3), 580-582.

RozonoéR, L.I. (1959) L. S. Pontryagin's Maximum Principle in the Theory of Optimal Systems, Part I-III (in Russian). Avtomatika $i$ Telemekhanika 20, 1320-1334, 1441-1458, 1561-1578. Translated into English: Automation and Remote Control 20, 1288-1302, 1405-1421, 15171532.

Sussmann, H.J. and Willems, J.C. (1997) 300 Years of Optimal Control: From the Brachistochrone to the Maximum Principle. IEEE Control Systems Magazine 17, 32-44.

Weierstrass, K.T.W. (1879) Vorlesungen über Variationsrechnung. In: R.E. Rothe, ed., Gesammelte Werke von Karl Weierstrass 7. Akademische Verlagsgesellschaft, Leipzig, 1927. Reprints: Georg Olms, Hildesheim, 1967; Johnson, New York, 1967.

Valentine, F.A. (1937) The Problem of Lagrange with Differential Inequalities as Added Side Conditions. PhD Thesis, University of Chicago. 


\section{Picture credits:}

Magnus R. Hestenes: Thanks to Dr. Ronald F. Boisvert, Mathematical and Computational Science Division of the Information Technology Laboratory at the Natonal Institute of Standards and Technology in Gaithersburg.

Rufus P. IsaAcs: Thanks to Prof. Dr. Michael H. Breitner.

Richard E. Bellman: http://en.wikipedia.org/wiki/Richard_E._Bellman.

Lev Semyonovich Pontryagin: http://www-groups.dcs.st-andrews.ac.uk/ nistory/PictDisplay/Pontryagin.html.

Vladimir Grigor'evich Boltyanskir: From Boltyanskii's former homepage at the Centro de Investigación en Matemáticas, Guanajuato, Mexico.

Revaz Valerianovich Gamkrelidze: Photo taken by the first author at the Banach Center Conference on 50 Years of Optimal Control in Będlewo, Poland, September, 2008. 
